\title{
Factors Affecting the Development of Colon Cancer and Electrical Stimulation of the Colon
}

\author{
Mohammad Neshatpour Esfahani 1,* \\ ${ }^{1}$ Felavarjan Branch, Islamic Azad University, Isfahan, Iran
}

Corresponding Author: Mohammad Neshatpour Esfahani, MSc, Islamic Azad University, Felavarjan Branch, Isfahan, Iran. Tel: +98-9378940821, E-mail: mansoore7070@gmail.com

Received May 24, 2017; Accepted July 12, 2017; Online Published August 29, 2017

\begin{abstract}
One cause of mortality in large countries of the world today is colon cancer. One main reason behind the development of colon cancer is constipation. Using electric shock, the colon can regain its motility. This electrical stimulation includes a series of electric arrays. Patients with constipation are categorized into two groups, apart from those who only show the disease symptoms. A patient whose rectum had lost its excitability after stroke was studied. The patient clearly benefited from electric stimulation treatment. Considering the fact that the biological feedback of this experiment is intense, the patient should cooperate. Electrical stimulation treatment is performed with a highly complex device. This treatment can be useful for many patients, especially those who suffer from impaired rectal contraction.

Keywords: Neoplasms; Constipation; Electric Stimulation

Citation: Neshatpour Esfahani M. Factors affecting the development of colon cancer and electrical stimulation of the colon. Int J Med Rev. 2017;4(3):70-75. doi: 10.29252/ijmr-040303.
\end{abstract}

\section{Introduction}

Around $70 \%$ of people in developed countries across the world suffer from colon-related diseases, including constipation. Such diseases can have various symptoms and complications for the patient. Chronic constipation can develop into various diseases over time for the patient, including hemorrhoid, urinary tract infection, ovarian problems in middle-aged women, enuresis in children, and, more importantly, colon cancer. ${ }^{1}$ Constipation means having labored defecation less than three times a week. This disorder in the U.S. made 2.5 million people visit a physician between 1959 and 1986 . Naturally, this disease can impose staggering economic costs upon both developed and developing countries of the world. ${ }^{2}$ When first dealing with patients who have constipation, several issues should be taken into account. First, the patient's symptoms and statements should be matched with definitions. In some cases, the patient is not indeed a patient, but mistakenly expresses constipation. For example, around $30 \%$ of patients who visited the clinic at Shariati Hospital, Tehran, within a specific period of time had no constipation. ${ }^{3}$ Slow transit constipation (STC) is a tolerable subset of diseases in children. The main cause is phosphorus deficiency in axons and, generally, hypo-functionality of axons that stretch to the colon muscles. Electrical stimulations can influence this mechanism. During the initiation of internal studies, it was found that all individuals show the same response to electrical stimulations. Studies have suggested that in treating children with STC, stimulations applied constantly can be effective. ${ }^{4}$ The current study investigated the effect of electricity on colon function. Discharge of the colon contents is usually controlled by the external sphincter of the rectum, which composed of smooth muscles. One treatment for colon diseases is colostomy, in which the terminal part of the colon is removed for different pathological reasons, and the control on the terminal part is lost. The healthy colon is attached to the abdominal wall, and the surrounding colon is attached through stoma. If there is a method instead of normal discharge, one can prevent undesired discharges. During experiments conducted by Sevcencu et al., artificial contractions developed in several regions of rats' colons. Although studies have shown that the digestive tract can be contracted with a wide range of electrical stimulations, stimulation of the colon with 0.2 $100 \mathrm{~ms}, 50-10 \mathrm{~mA}$, and $3-1670 \mathrm{~Hz}$ has achieved the best response. High amplitudes and over-lengthiness of pulses can cause thermal or electrolyte damage around

Copyright (C) 2017 The Author(s). This is an open-access article distributed under the terms of the Creative Commons Attribution License (http://creativecommons.org/licenses/by/4.0), which permits unrestricted use, distribution, and reproduction in any medium, provided the original work is properly cited. 
electrodes. Furthermore, within the stated ranges, if the minimum possible value is employed, less energy is used, and thus, a better system for electrical stimulation is developed. Another question that may arise in this study is whether or not neural responses are involved in the course of this experiment. During electrical stimulation, muscles, enteric nerves, or even both may be stimulated. Electrical stimulation of muscles always results in contraction, while electric stimulation of enteric nerves leads to the development of relaxation or of contractions along the colon, although this response is dependent on the properties of the stimulated neuron. ${ }^{5}$

\section{Colon}

Discharge of the colon contents is usually controlled by the external sphincter of the rectum, with this sphincter being composed of smooth muscles. ${ }^{5}$

\section{Colon Diseases}

One treatment for colon diseases is colostomy. In this procedure, the terminal part of the colon is removed due to several pathological reasons, and control on the terminal part is lost. ${ }^{5}$ Constipation refers to the prolonged residence of materials in the colon. There are different types of constipation. One type is mild constipation, which refers to a medical treatmentresistant condition. Current treatments are limited to modifying the diet and employing laxatives. Treatments such as bio feeds are used to manage this disease. A second type of constipation is the slow movement of colon contents due to motility disorders, and a third type is called pelvic floor dyssynergia. There is another subgroup of this condition which is caused by the diminished or even absence of sensation of the presence of feces. This disease may be caused by pathophysiological factors of the rectal opening in creating neural messages. All of the above-mentioned conditions are generally referred to as constipation. ${ }^{6}$

\section{Electrical Stimulation}

Electrical stimulation can be useful for pathologies and diseases such as constipation and slow motility of digestive tract contents. Various studies have been performed on different electrical simulation methods, and successful results for the stomach have been reported. Artificial gastric lavage is a suitable substitute for the use of pro-kinetics or surgical methods. This study developed artificial contractions in several regions of rats' colons ${ }^{5}$. In one case study, a 25-year-old woman who had suffered from constipation for 10 years visited a doctor. She had used conventional treatments such as laxatives, teas, and a high-fiber diet, but nothing had proven effective. She had even taken very potent medications, but again no response had been achieved. After a great deal of suffering, she despaired of ever being cured and attempted suicide. When she was transferred to the hospital, she underwent warm water therapy. Electrical stimulation treatment may be performed by stimulating electrodes externally or inside the sacral nerve or rectum using implanted devices. In previous studies, stimulation was performed through electrodes implanted by surgery. ${ }^{6}$

\section{Screening Recommendations}

People above the age of 50 years are at risk for colon cancer. In screening experiments, it has been observed that people between the ages of 40 and 49 years have a low incidence of this disease. These results suggest that screening should be performed for people aged 50 years or older. In screening, some important points should be considered, including people's trust in methods and physicians, and the assurance that screening can actually prevent incidence of disease. ${ }^{7}$

\section{Factors Affecting Electrical Stimulation}

To investigate the motility of contents inside the lumen by local coordinated contractions, an object was placed inside the colon. Stimulation was performed by an electrode array. This experiment was performed on a total of 40 male rats with weights of 420-490 g which were studied and verified by a Danish animal husbandry committee. The animals had complete access to chow and tap water. After induction of anesthesia, the experiment was initiated with a series of substances including fentanyl $0.21 \mathrm{mg} / \mathrm{kg}$, fluanisone $6.75 \mathrm{mg} / \mathrm{kg}$, and midazolam $3.3 \mathrm{mg} / \mathrm{kg}$. Thereafter, the abdomen was opened and the colon became accessible. After that, the feces were removed with mild pressure from the hole which had been created in the top part of the rectum. Almost $5 \mathrm{~mm}$ after the bending, a second hole was created to place from $\mathrm{O}$ point to $\mathrm{B}$ point (length $=22$ $\mathrm{mm}$, diameter $=8 \mathrm{~mm}$, and weight $=1.5 \mathrm{~g}$ ) composed of a plastic material, and its shape was in line with the fecal bullets found in that part of the colon. The distance between the two holes was $47.4 \mathrm{~mm} .^{8}$

\section{Analyzing Factors Affecting Stimulation}

e1 to e 5 represent the electrodes implanted in the colon; s1 to $s 4$ show the parts between the electrodes;

p1 to 44 indicate the stages of development and the course of the procedure;

s1 to $s 4$ suggest the stages;

fc indicates the displacement exchanger including ferrite kernels

$c=$ coil slide

$\mathrm{r}=$ resistance

$\mathrm{v}=$ voltage

$\mathrm{f}=$ frequency

Part B represents the recording of p1-4 displacement in response to the sessions of electrical stimulation (s1-4) (Figure 1).

The electrodes were made of multipurpose stainless steel insulated with Teflon. The insulator in the tip of the wire had been withdrawn by $8 \mathrm{~mm}$. using a thin injection needle in active regions, five electrodes of the wire were placed directly, vertical to the longitudinal axis of the 
colon. Based on the length of the studied animal's colon, distances between the two electrodes varied between 7 and $10 \mathrm{~mm}$. In the first experiment on 10 rats, the numerical model of electrical stimulation was developed by $10 \mathrm{~mA}, 0.3 \mathrm{~ms}$, and $40 \mathrm{~Hz}$, which was connected to arrays of electrodes using an electrical enclosure. In the first stimulation (S1), electrodes e1 and e2were used, which pushed OB from S1 to S2. Along the stimulation of the S1 part, e1 was the cathode and e2 was the anode. Stimulation continued until the bolus stopped after motion.

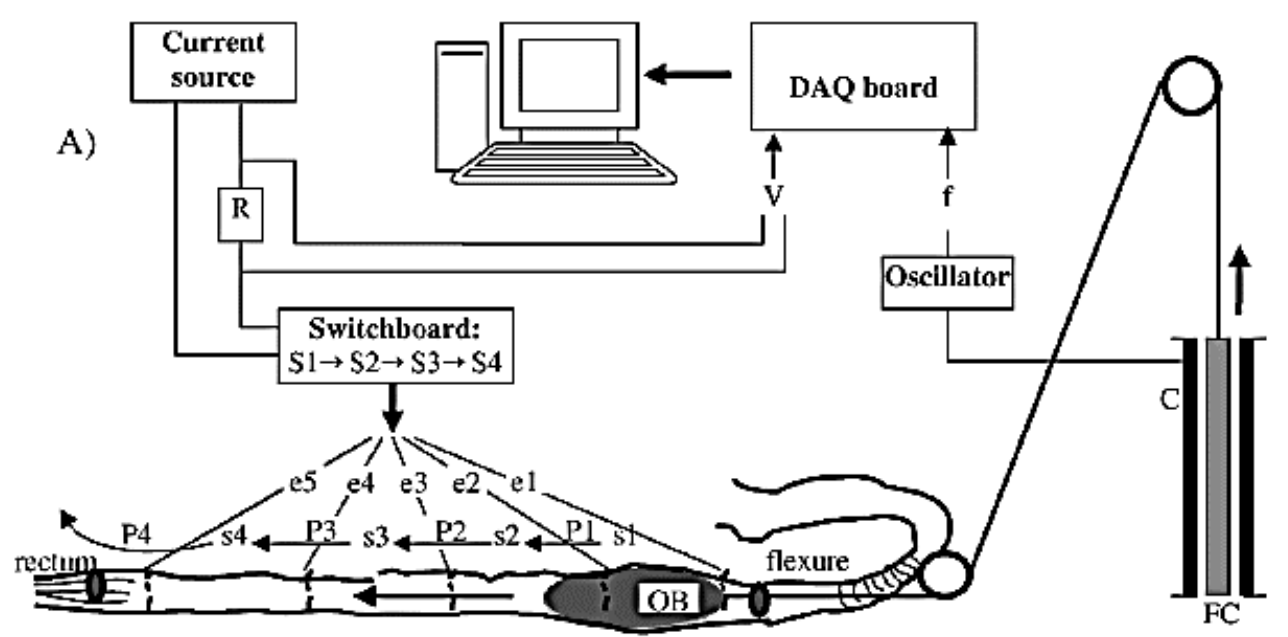

Figure 1. Discharging the Colon by Electrical Stimulation in Rats

After completion of the stimulation of the P1 part, the second stimulation session (which was performed for P2) was initiated. This stimulation was conducted by e2 and $\mathrm{e} 3$ electrodes. At this time, the e 2 electrode changed in polarity from a positive pole (anode) to a negative pole (cathode). The following stages continued in the same way until the fourth stage was completed, whereby the $\mathrm{OB}$ bolus was removed from the terminal hole. According to visual control, each session of stimulation continued until completion of the induction force. With the same stimulation pattern, the effects of different combinations of the duration of the amplitude pulse and frequency of the stimulation were examined in the 15 other cases. Two to four series of stimulations were performed on each animal with an interval of around 7 min. The following combinations were used from the parameters of the utilized pulses (represents the utilized pulses).

Finally, the cholinergic and nitro genic block experiments in the biological pathways were conducted. In order to understand which biological pathways affect the experiments in the body, stimulations were performed on the colon $(5 \mathrm{~mA}, 0.3 \mathrm{~ms}, 10 \mathrm{~Hz}) 15 \mathrm{~min}$ after the injection of L-NAME $(10 \mathrm{mg} / \mathrm{kg})$, atropine (16 $\mathrm{mg} / \mathrm{kg}, 5)$. The same saline volume was injected and stimulation was applied with the same method. OB displacement was measured by a custom-made device. The mechanical part of the displacement exchanger consisted of a ferrite kernel (1.8 $\mathrm{mg}$ and $30 \mathrm{~mm}$ in length) with a connection to OB. The ferrite kernel could slide with no friction to the coil which was part of an oscillator. Along the displacement, OB determined the frequency. It is a device that reflects the extent of displacement. As the ferrite kernel is shorter than the descending colon (30 mm versus $47 \mathrm{~mm}$ ), only P1-P3 stages were studied. Nevertheless, this method represents $65 \%$ of the entire length of the descending colon. The first response after initiation of the electrical stimulation session was a shortening of the descending region of the colon as a single region. Immediately after contraction of longitudinal muscles, contraction of circular muscles began. Serial and continuous contractions of circular muscles caused the OB kernel to move forward. When the stimulation was applied to the colon with $4 \mathrm{~mA}, 0.3$ $\mathrm{ms}$, and $40 \mathrm{~Hz}$, the kernel was unable to traverse more than half of the length of the descending colon. Accordingly, this group was not further analyzed. Across all of the other stimulations, the OB kernel traversed the complete length of the colon and left it from the end. Since the distance between the points was always the same, only the delay time and displacement rate were considered to find the best combination of parameters.

\section{Effects of Continuation of Electrical Stimulation}

The delay in the colon when the pulse was applied for 0.1 $\mathrm{ms}$ more was far longer than the time observed when $0.15,0.2$, and $0.3 \mathrm{~ms}$ were observed. This is a significant difference suggesting that the longer the duration of the pulse, the shorter the delay in development of force is. Comparing the results of applying the maximum electric force $(10 \mathrm{~mA}, 0.3 \mathrm{~ms}, 40 \mathrm{~Hz})$ and applying the minimum electric driving force $(5 \mathrm{~mA}, 0.3 \mathrm{~ms}, 10 \mathrm{~Hz})$, a time delay was observed, though no change in the rate was observed. $^{8}$ Colonoscopy is recommended to be performed once every 10 years. In half of the people with colon cancer in the proximal part of the colon, no lesion has been observed in the left part. Using colonoscopy, which gives the possibility of observing the entire colon 
and identifying and removing polyps, the rate of mortality due to colon cancer has diminished significantly. Nevertheless, colonoscopy has some disadvantages. First, it is not possible to see the entire colon at all times, and up to $6 \%$ of the polyps inside the colon may not be observed. For this reason, it is recommended that after this screening test, the FOBT test be performed as well. In the colonoscopy test, the patient experiences considerable pain and labor, yet colonoscopy is considered one of the best prevention methods, and it is recommended to be performed once every 10 years. This time range is recommended because according to research, an adenomatous polyp does not change significantly enough to develop into colon cancer within five years. ${ }^{7,8}$ The end of a healthy colon is attached to the abdominal wall, while the surrounding parts of the colon are attached by stoma ${ }^{9}$. Problems in the motor nerve of the colon prevent the development of force. Electrical stimulation is a kind of driving force. Scientists have attempted to find a solution to these issues to recover motion and the ability to perform natural and daily routines. The studies have been trying to optimize contraction parameters. ${ }^{5}$

\section{Barium Enema with Air Injection}

This method is recommended for those who do not like to have a colonoscopy performed. The success rate of this method is $85 \%$, while colonoscopy offers $95 \%$. In this method, $53 \%$ of polyps $6-10 \mathrm{~mm}$ in size can be observed. With this method, the chance of finding polyps is slimmer, and performing a biopsy is not possible. However, it is possible to observe waste particles as polyps. In any case, it is a diagnostic method with which the entire colon can be observed, and this procedure can be performed in all healthcare centers.

\section{Genetic Diseases in the Development of Colon Cancer} Familial polyposis cancer is a genetic disease which is transferred as a dominant gene and is a result of mutation in APC genes. People suffering from this disease are $100 \%$ at risk of developing colon cancer. The average age at which polyps develop is 16 years, while the average age at which colon cancer develops is 39 years. These patients often have more than 100 adenomatous polyps. $^{7}$

\section{Following up with Patients with Colon Cancer}

Patients who have undergone surgery for colon cancer should be constantly screened and followed up so as to determine possible incidence of synchronous tumor. If during colon surgery, got obstructed, a colonoscopy should be performed six months later. However, if a colonoscopy was performed on the patient sometime during the course of treatment before surgery, the next one should be conducted three years later; and if the results were normal, the next colonoscopy should be performed five years later. Research has shown that colonoscopy follow-up following surgery is not very successful. In cases were a tumor recurrence is observed, some conditions preclude surgery, which causes infiltration into the abdominal cavity. Annual colonoscopy has not shown disease relapse in most cases, while these issues can be followed up with other diagnostic methods, such as CXR and CT scan ${ }^{7}$. First, constipation should be diagnosed using the available diagnostic methods. Among the current methods are Barium enema, colonoscopy, scintigraphy, manometry, etc. ${ }^{10}$

\section{Existence of DNA in Feces}

Since colon cancer develops through an alteration in the DNA of the tissue of the colon and colon tissue cells can be tracked through feces, the incidence of colon cancer can be tracked by analyzing the DNA of dead cells in feces. Although this method is being performed in some laboratories and has also offered promising results, further evidence should be collected in this regard. With the advances in novel technologies, the progress in human genomic projects, and new information about the genetic polymorphisms involved in colon cancer that have come to light, new methods are certain to be developed in the future. Nevertheless, the lives of many current patients can be saved by maximizing usage of the facilities available and mitigating both financial costs and casualties in response to cancer and other diseases.

\section{Discussion}

This paper has discussed the general prevalence of constipation and functional constipation. The estimated prevalence of these conditions is $33.4 \%$ and $15.2 \%$, respectively, which is a higher percentage than those reported by most studies in Iran or in other countries. The prevalence of constipation has been reported to be $14.9 \%$ in Canada and $16.5 \%$ in North Korea and 1.4$37.0 \%$ in Iran. Recent studies have found that women suffer from constipation more than men. All of the stated symptoms are more frequent in women than in men. One reason for this difference may be that women express their disease symptoms more than men. Pare et al. in North America indicated that the incidence of constipation is twice as frequent in women as in men. The findings were also similar in the metropolises of Tehran and Isfahan, suggesting a higher frequency of constipation among women. Wald et al. stated that elevations in progesterone in the luteal stage is associated with digestion transit. Constipation and functional constipation are common in women above 50 years of age. However, such a relationship between age and extent of constipation and functional constipation has not been observed among men. Many studies have hypothesized that with aging, the amount of energy required by a person declines and food intake also diminishes, causing a reduction in the weight of feces and predisposing the person to constipation ${ }^{1,11}$. The first response after beginning the electrical stimulation session was the shortening of the descending region of the colon as a 
single region. Immediately after contraction of the longitudinal muscles, contraction of the circular muscles was initiated. Serial continuous contractions of circular muscles caused the OB kernel to move forward. When the stimulation was applied to the colon $(4 \mathrm{~mA}, 0.3 \mathrm{~ms}$, and $40 \mathrm{~Hz}$ ), the kernel was not able to traverse more than half the length of the descending colon. Since the distance between the points was always the same, the delay time and displacement rate were taken into account to find the best combination of parameters. The delay in the column when the pulse of $0.1 \mathrm{~s}$ was applied was greater than when pulses of $0.15,0.2$, and $0.3 \mathrm{~ms}$ were applied. This difference is significant, suggesting that the longer the pulse duration, the shorter the delay will be in the creation of the force. When the maximum electrical force $(10 \mathrm{~mA}, 0.3 \mathrm{~ms}, 10 \mathrm{~Hz})$ was applied and compared with the application of the minimum electric driving force $(5 \mathrm{~mA}, 0.3 \mathrm{~ms}, 10 \mathrm{~Hz})$, a time delay was seen, but no change in rate was observed. The absence of any motion before that was due to the usage of fentanyl. Although the electrical stimulation applied across different occasions was the same, different responses were observed from the colon. There were significant differences in the length and rate of movement of colon contents due to alterations in the frequency, impulse duration, and amplitude variations. Although published papers have mentioned differences among colons across different species, this study found that differences can also exist between similar species and similar colons within a species. Considering the fact that motility is greater in the lower part soft the colon, they can change themselves with stimulation. Furthermore, if stimulation occurs in the upper parts of the colon, it is attenuated until reaching the lower parts, though this possible work needs reliability.

\section{Conclusions}

The current results suggest that this is the last valid result for the stimulation duration. A short stimulation period causes reduced energy consumption, which is an advantage in stimulation systems. Implant systems consume less energy and impose less damage to the tissues. Prolongation of stimulation from 0.1 to $0.2 \mathrm{~ms}$ causes the shortened retention time of colon contents due to increased stimulation power. However, these values for electrical stimulation are for currents above 7.5 $\mathrm{mA}$. For less than $7.5 \mathrm{~mA}$, the impulse stimulation time should be prolonged, which can be increased to $0.3 \mathrm{~s}$. The present study employed an impulse time of $0.3 \mathrm{~s}$. When $4 \mathrm{~mA}$ was used, only half of the colon was prepared for stimulation. In addition, the retention time of bolus in the colon increases. These stimulations can activate two types of nerves synergistically for the development of contractions, but this state occurs across different thresholds. All of the experiments were performed on anesthetized animals. One of the adverse effects of anesthesia in the course of this experiment was the effect of fentanyl which prevented the effect of peristalsis. On the other hand, the second dose may be followed by elevated levels of contractions in the colon muscles. This occurs through inhibition of the adrenergic effect of dopamine transfer. Despite such an inhibitory effect observed during anesthesia on animals being studied, electrical stimulation could still cause contraction. It seems that electrical stimulation can prevent this inhibition from happening. It seems that at times other than anesthesia, one can achieve similar results by developing weaker stimulations. The results obtained from implantable electrodes in swine colon confirm this. On the other hand, the activation of cholinergic neurons causes contraction in the motor part. NO released from the nitro genic terminals causes relaxation in the receiving part. Direct activation of muscles needs very long impulses of around $50 \mathrm{~ms}$, whereas activation of muscles by neurons needs only about $1 \mathrm{~ms}$. this means that the entire colon wall will be innervated.

\section{Acknowledgments}

The authors wish to express their appreciation for the assistance of Professor Amin Mahnam (medical engineering, Isfahan University), Professor Tahmasb Shahgholian (physiology specialist at Islamic Azad University, Sharekord), Dr. Mohammad Hosssein Mirhosseini, and Dr. Mansoureh Sadeghi, and all subjects who participated in this study.

\section{Conflicts of Interests Disclosure}

None.

\section{Funding}

None.

\section{References}

1. Amirbaigy M, Salman Roughani H, Hosaini N, Bootorabi Z. Assessment of colon transit time in idiopathic chronic constipation. SSU J. 2006;14(3):20-4.

2. Drossman DA, Sandler RS, Mckee DC, Lovitz AJ. Bowel Patterns among Subjects Not Seeking Health-Care - Use of a Questionnaire to Identify a Population with Bowel Dysfunction. Gastroenterology. 1982;83(3):529-34.

3. Nam YS, Pikarsky AJ, Wexner SD, Singh JJ, Weiss EG, Nogueras JJ, et al. Reproducibility of colonic transit study in patients with chronic constipation. Dis Colon Rectum. 2001;44(1):86-92. pmid: 11805568.

4. Hutson JM, Dughetti L, Stathopoulos L, Southwell BR. Transabdominal electrical stimulation (TES) for the treatment of slow-transit constipation (STC). Pediatr Surg Int. 2015;31(5):445-51. doi: 10.1007/s00383-015-3681-4. pmid: 25672282.

5. Sevcencu C, Rijkhoff NJ, Gregersen H, Sinkjaer T. Electrical stimulation to induce propulsive contractions in the porcine descending colon. Artif Organs. 2005;29(3):246-9. doi: 10.1111/j.1525-1594.2005.29045.x. pmid: 15725227.

6. Chang HS, Myung SJ, Yang SK, Yoon IJ, Kwon OR, Jung HY, et al. Functional constipation with impaired rectal sensation improved by electrical stimulation therapy: report of a case. Dis Colon Rectum. 2004;47(6):933-6. doi: 10.1007/s10350004-0521-3. pmid: 15073666.

7. Zali M. [Colon Cancer, Early Diagnosis and Prevention Strategy]. Iranian J Surg. 2004;12(31):1-13.

8. Ansari R, Mahdavinia M, Sadjadi A, Nouraie M, Kamangar $F$, Bishehsari $F$, et al. Incidence and age distribution of 
colorectal cancer in Iran: results of a population-based cancer registry. Cancer Lett. 2006;240(1):143-7. doi: 10.1016/j.canlet.2005.09.004. pmid: 16288832 .

9. Sevcencu C, Rijkhoff NJ, Sinkjaer T. Colon emptying induced by sequential electrical stimulation in rats. IEEE Trans Neural Syst Rehabil Eng. 2005;13(4):516-23. doi: 10.1109/TNSRE.2005.859358. pmid: 16425834.
10. Albert KM. Open access: implications for scholarly publishing and medical libraries. J Med Libr Assoc. 2006;94(3):253-62. pmid: 16888657.

11. Office of Deputy Minister for Health Center for disease control MoHaME, Islamic Republic of Iran. Iranian Annual National Cancer Registration Report. Iran: 2007. 